\title{
Conductas alimentarias de riesgo y correlatos psicosociales en estudiantes universitarios de primer ingreso con sobrepeso y obesidad
}

\author{
Claudia Unikel Santoncini, ${ }^{1}$ Concepción Díaz de León Vázquez, ${ }^{2}$ José Alberto Rivera Márquez ${ }^{3}$
}

Artículo original

\section{ABSTRACT}

\section{Introduction}

The beginning of college studies involves important stress, which is related to an increase in the incidence of depression, anxiety, alcohol abuse and DEB.

\section{Objective}

To analyze the relationship between DEB and nutritional status measured with $\mathrm{BMI}$, its association with demographic and psychological variables, and the interaction with BMI.

\section{Method}

A sample of 892 college students in their freshman year $(502$ females and 390 males) ( $X_{\text {age }}=19.7 ; S D=2.9$ ) was studied. Weight and height were measured, and validated questionnaires were used.

\section{Results}

The prevalence of DEB was $6.1 \%$ in females and $4.1 \%$ in males. The prevalence of overweight and obesity in females was $22.6 \%$, and $7.4 \%$ and $26.6 \%$ and $9.3 \%$ in males, respectively. Among women and men with obesity $10.8 \%$ and $11.1 \%$ showed high-DEB, whilst among overweight women and men $13.2 \%$ and $3.8 \%$ showed high$D E B$, respectively. $B M I$ increases the risk for DEB 1.6 times among women $(p<0.05), 1.4$ times among men $(p>0.05)$. There was no interaction found between $\mathrm{BMI}$ and the other variables.

\section{Discussion and conclusion}

The variables related with body image increase the risk of DEB significantly $(p<0.05)$ in both sexes. Depressive symptoms, low self-steem increase the risk among women, and socioeconomic status and study area increase the risk of DEB among men. The specific research of $D E B$ among males is highly recommended to avoid the under registration of cases.

Key words: Disordered eating behaviors, body mass index, selfsteem, youth.

\section{RESUMEN}

\section{Introducción}

El comienzo de los estudios universitarios es un estresor importante para los jóvenes que se asocia con un incremento en la incidencia de depresión, ansiedad, abuso de alcohol y CAR.

\section{Objetivo}

Analizar la relación entre CAR e IMC, su asociación con variables demográficas y psicológicas y la interacción con el IMC.

\section{Método}

En una muestra de 892 estudiantes universitarios de nuevo ingreso (502 mujeres y 390 hombres) $\left(X_{\text {edad }}=19.7 ; D E=2.9\right.$ ) se midió peso y estatura y se utilizaron cuestionarios validados.

\section{Resultados}

La prevalencia de CAR fue de $6.1 \%$ en mujeres y de $4.1 \%$ en hombres. La prevalencia de sobrepeso y obesidad en mujeres fue de $22.6 \%$ y de $7.4 \%$, y en hombres fue de $26.6 \%$ y de $9.3 \%$, respectivamente. Las mujeres y los hombres con obesidad $10.8 \%$ y $11.1 \%$ presentaron CAR de frecuencia alta, y las mujeres y hombres con sobrepeso $13.2 \%$ y $3.8 \%$, respectivamente. En las mujeres el IMC aumenta el riesgo de CAR 1.6 veces $(p<0.05)$ y en los hombres 1.4 veces $(p>0.05)$. No se encontró interacción del IMC en la asociación de CAR con el resto de las variables.

\section{Discusión y conclusión}

En ambos sexos, las variables relacionadas con la imagen corporal aumentan el riesgo de CAR significativamente $(p<0.05)$. Los síntomas depresivos, la baja autoestima y el IMC aumentan el riesgo entre las mujeres, y el deseo por la musculatura, nivel socioeconómico y el área de estudio entre los hombres. Se recomienda llevar a cabo una investigación específica sobre CAR en varones, para evitar el subregistro de casos.

Palabras clave: Conductas alimentarias de riesgo, índice de masa corporal, autoestima, jóvenes.

\section{Dirección de Investigaciones Epidemiológicas y Psicosociales, Instituto Nacional de Psiquiatría Ramón de la Fuente Muñiz, Ciudad de México. \\ 2 Departamento de postgrado, Universidad Autónoma Metropolitana, Unidad Xochimilco, Ciudad de México.}

Departamento de Atención a la Salud, Universidad Autónoma Metropolitana, Unidad Xochimilco, Ciudad de México.

Correspondencia: Claudia Unikel Santoncini. Dirección de Investigaciones Epidemiológicas y Psicosociales, Instituto Nacional de Psiquiatría Ramón de la Fuente Muñiz, Calzada México-Xochimilco 101, San Lorenzo Huipulco, 14370, Ciudad de México. Tel: 554160 - 5160 . E-mail: unikels@imp.edu.mx

Recibido primera versión: 18 de junio de 2015. Segunda versión: 21 de julio de 2015. Aceptado: 6 de mayo de 2016. 


\section{INTRODUCCIÓN}

Los problemas de salud mental pueden incrementar su incidencia en los jóvenes que comienzan sus estudios universitarios, dado que experimentan el estrés de una decisión clave para su vida, un mayor grado de independencia y autonomía; algunos de ellos se han separado de su familia para estudiar en otra ciudad. Esta nueva condición puede influir en sus hábitos de alimentación, desempeño escolar y desarrollo emocional. ${ }^{1-3}$

El sobrepeso y la obesidad son considerados problemas de salud porque están asociados con enfermedades crónico-degenerativas y deterioro en la funcionalidad. ${ }^{4}$ En México, la Encuesta Nacional de Salud y Nutrición 2012 (ENSANUT-2012) ${ }^{5}$ reporta que $23.0 \%$ de las mujeres adolescentes (11-19 años) tienen sobrepeso y $12.0 \%$ tienen obesidad. Mientras que $19.0 \%$ y $14.0 \%$ de los hombres de este mismo grupo de edad presentan sobrepeso y obesidad respectivamente. Entre las mujeres de 20 a 49 años de edad se encontró que $35.0 \%$ presentan sobrepeso y la misma proporción presenta obesidad. Los hombres mayores de 20 años presentan sobrepeso y obesidad en una frecuencia de $42.0 \%$ y $26.0 \%$ respectivamente.

En la actualidad está muy generalizado en la población el deseo por una figura corporal delgada, principalmente en las mujeres, y de una figura mesomórfica en los hombres. Las personas con sobrepeso y obesidad se enfrentan al estigma relacionado con la figura y el peso corporal, el cual proviene de la creencia de que las personas con obesidad son diferentes en términos conductuales y de personalidad, que tienen poca fuerza de voluntad y son poco atractivos. ${ }^{6}$ Suelen ser víctimas de comentarios negativos que afectan la autoestima y que se asocian con la adopción de conductas alimentarias de riesgo (CAR). Estas últimas, por su parte, se ponen en práctica con la intención de reducir el peso corporal, pero de manera paradójica colocan a los sujetos en una situación de mayor ganancia de peso. ${ }^{7}$

El índice de masa corporal (IMC) se ha asociado con las CAR en mujeres y hombres con sobrepeso y obesidad. ${ }^{8}$ Las CAR son conductas características de los trastornos de la conducta alimentaria (TCA) que se presentan en menor frecuencia e intensidad. Entre ellas se encuentran los atracones y las conductas restrictivas y purgativas, y junto con el sobrepeso y la obesidad se han considerado como factores de riesgo para el desarrollo de TCA. ${ }^{9}$

En México, Palma et al., ${ }^{10}$ encontraron que $6.6 \%$ y $6.3 \%$ de los jóvenes entre 10 y 19 años, con sobrepeso y obesidad, respectivamente, presentan alguna CAR, en comparación con $2.6 \%$ de los jóvenes que presentan peso normal ( $p<0.0001)$. En la misma muestra, se encontró que entre los jóvenes de hogares cuyo nivel socioeconómico era más alto, existía un riesgo más elevado de presentar CAR (OR: 2.05, 95\% IC: 1.52 - 2.75).

Los estudios realizados en poblaciones de universitarios en México han mostrado la presencia de CAR en porcentajes que fluctúan desde $7.9 \%{ }^{11}$ hasta $18.9 \%{ }^{12}$ en mujeres, y de
$4.2 \%{ }^{11}$ hasta $13.0 \%{ }^{13}$ en hombres. En otros países de América Latina se han encontrado porcentajes entre $8.3 \%{ }^{14}$ en un estudio realizado en mujeres estudiantes universitarias en Brasil y hasta $44.1 \%$ en mujeres estudiantes de medicina en Colombia. ${ }^{15}$ En varones, los porcentajes varían de $1.9 \%$ en estudiantes venezolanos hasta $9.6 \%$ en estudiantes colombianos. ${ }^{16}$ En un estudio en el que participaron 935 universitarias brasileñas de nuevo ingreso, se encontró que las estudiantes con IMC normal presentaron prevalencias más altas de CAR en comparación con las que presentaron bajo peso y sobrepeso u obesidad: $10.0 \%, 2.9 \%$ y $3.9 \%$, respectivamente. ${ }^{14}$

En la actualidad, el ideal de atractivo físico en los hombres es el de un cuerpo mesomorfo, en el cual es posible observar una musculatura marcada en ausencia de grasa corporal. En los medios de comunicación se ha incrementado la exposición de este tipo de imágenes, lo que pone al cuerpo de los varones en condición de ser objetivizado de la misma forma como ha sucedido con los cuerpos femeninos; es decir, como objetos para ser vistos y examinados siempre desde la perspectiva de la apariencia. Cuando los sujetos interiorizan una posición de observadores respecto a su propio cuerpo, surge una forma de autoconciencia que se caracteriza por la auto-monitorización. Esto puede generar vergüenza y ansiedad relacionada con el cuerpo y como consecuencia, la aparición de algún TCA. ${ }^{17}$ Desde la perspectiva de Cafri et al., ${ }^{18}$ existe una asociación entre el deseo por alcanzar una figura corporal mesomórfica y el desarrollo de CAR, razón por la cual han propuesto el estudio del deseo de la musculatura (DM) como un factor que incrementa la frecuencia de CAR entre los varones.

Otros factores asociados con el desarrollo de TCA, son la interiorización del ideal estético de la delgadez (IIED), la insatisfacción corporal (IC), la autoestima y los síntomas depresivos (SD). Se ha encontrado, en estudiantes universitarios, que la comorbilidad psiquiátrica se incrementa en presencia de CAR. ${ }^{19}$ Los síntomas depresivos son algunos de los factores de riesgo con mayor potencia para predecir TCA, según lo reportado por Jacobi et al. ${ }^{20}$ La presencia de la IIED y de IC también ha sido reportada en estudios longitudinales como factores de riesgo para TCA, ${ }^{21}$ así como la presencia de autoestima baja antes de la aparición de este tipo de psicopatología. ${ }^{22}$

Este trabajo tiene varios objetivos; analizar de manera separada para hombres y mujeres: 1) la relación entre CAR e IMC, 2) la interacción del IMC en la asociación de CAR con variables demográficas y psicológicas y 3) la asociación de CAR con todas las variables de estudio.

\section{MÉTODO}

\section{Muestra}

Fueron invitados a responder una encuesta todos los estudiantes de primer ingreso al ciclo escolar de otoño de 2012 
de la Universidad Autónoma Metropolitana, Unidad Xochimilco (UAM-X), en la Ciudad de México. Previamente a la aplicación de la encuesta, se pidió a los sujetos su consentimiento por escrito, indicando la participación voluntaria y la confidencialidad de la información. Los criterios de inclusión fueron ser alumno de primer ingreso a la UAM-X en el período de estudio y aceptar la participación voluntaria; los criterios de exclusión fueron el no aceptar participar en el estudio, no concluir la encuesta, y, en el caso de las mujeres, estar embarazada. En la figura 1, se muestra la inclusión de los sujetos en la muestra, la cual quedó constituida por 892 estudiantes (502 mujeres y 390 hombres) que correspondió a una tasa de respuesta de $51.8 \%$ de la población de nuevo ingreso inscrita a la universidad durante el período de observación.

\section{Instrumentos}

La encuesta incluyó preguntas sobre datos demográficos: edad en años cumplidos, sexo y carrera. Se utilizó el Índice de la Asociación Mexicana de Agencias de Investigación de Mercados y Opinión Pública, Índice AMAI-Regla 10x6 (en adelante, índice AMAI) para estimar el nivel socioeconómico (NSE). ${ }^{23}$ El índice AMAI consta de cinco categorías, donde "A/B" representa el NSE más alto y "E" la categoría más baja de esta variable. Para obtener la frecuencia de CAR se aplicó el Cuestionario Breve para medir Conductas Alimentarias de Riesgo (CBCAR), ${ }^{24}$ utilizando dos puntos de corte: 1) riesgo moderado, puntuación entre 7 y 10 y 2) riesgo alto, puntuación $>10$. Este cuestionario cuenta con valores de sensibilidad y especificidad de 0.81 y 0.78 respectivamente, con un valor predictivo positivo de $0.38 \mathrm{y}$ un valor predictivo negativo de 0.96 .

Se incluyó el Cuestionario de Actitudes hacia la Figura Corporal $^{25}$ que mide la IIED, ante un puntaje igual o mayor

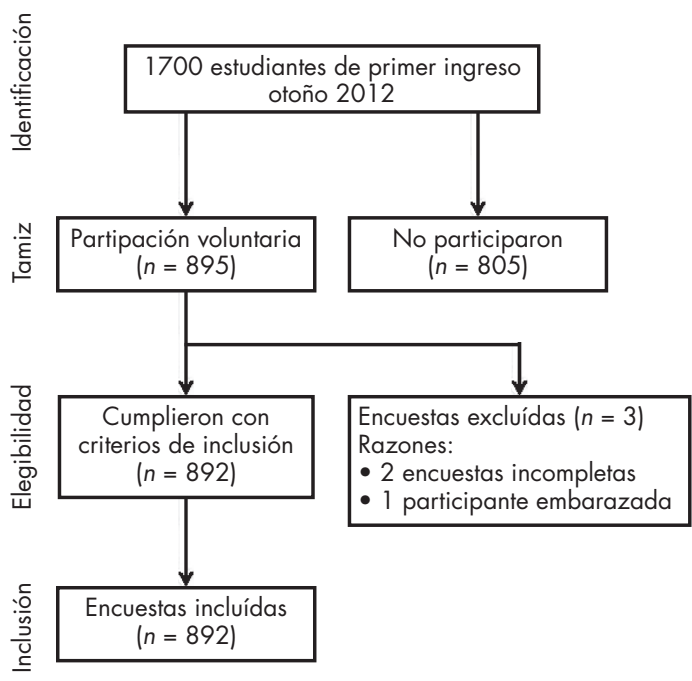

Figura 1. Diagrama de flujo para la selección de la muestra. a 37, con valores de sensibilidad y especificidad de 0.80 en ambos casos, con un valor predictivo positivo de 0.43 y un valor predictivo negativo de 0.96 .

Se utilizó el Cuestionario de Autoestima de Pope, McHale y Craighead, ${ }^{26}$ modificado por Unikel y Gómez Peresmitré, ${ }^{27}$ cuyas puntuaciones más altas indican menor autoestima. Este cuestionario se divide en dos subescalas de cinco y cuatro ítems, con un valor de confiabilidad alfa de Cronbach de 0.86 y 0.67 respectivamente, que explican el $59.6 \%$ de la varianza total.

La sintomatología depresiva (SD) se evaluó con la versión revisada de la Escala de Depresión del Centro de Estudios Epidemiológicos CESD-R, ${ }^{28}$ que consta de 35 reactivos con cuatro opciones de respuesta para registrar la presencia de SD durante las dos últimas semanas (escasamente 0-1 días, algo 1-2 días, ocasionalmente 3-4 días, la mayoría 5-7 días, casi diario 10-14 días). La validación en adolescentes mexicanos mostró características psicométricas adecuadas $(\alpha=0.93)$ y una estructura en seis factores $($ varianza total $=54.6 \%) .{ }^{29}$

El Cuestionario de Deseo por la Musculatura de McCreary (DM), ${ }^{30}$ validado en población mexicana por Escoto et al., ${ }^{31}$ cuenta con 15 preguntas con seis opciones de respuesta tipo Likert. Esta escala cuenta con un alfa de Cronbach total de 0.86 , se divide en tres factores que explican el $45.64 \%$ de la varianza total con valores de consistencia interna de 0.88, 0.77 y 0.68 .

Además, se empleó la Escala análoga de figuras corporales de Stunkard, Sorensen y Shulsinger, ${ }^{32}$ modificada por Acosta y Gómez, ${ }^{33}$ para medir la IC, la cual muestra una confiabilidad test-retest de 0.89 y una correlación significativa de 0.62 respecto a la subescala de insatisfacción corporal del Eating Disorders Inventory.

Así mismo, se incluyeron preguntas sobre el tiempo en minutos dedicados a realizar actividad física, cuyo parámetro de comparación fue la Guía de Actividad Física para Americanos (2008) $)^{34}$ y se preguntó sobre la carrera en la que estaban inscritos para formar las mismas categorías de áreas de estudio propuestas por la universidad: Ciencias y Artes para el Diseño (CAD), Ciencias Sociales y Humanidades (CSH) y Ciencias Biológicas y de la Salud (CBS).

Finalmente, se obtuvieron mediciones antropométricas de peso registrado en kilogramos y talla en metros, para calcular el IMC $\left(\mathrm{kg} / \mathrm{m}^{2}\right)$, el cual fue clasificado según los criterios de la Organización Mundial de la Salud: ${ }^{4}$ desnutrición $\leq 18.4$, normal de 18.5 a 24.9 , sobrepeso de 25 a 29.9 y obesidad $\geq 30 .{ }^{35}$ La talla se midió con cuatro estadímetros Seca modelo 206, con el sujeto de espaldas a la pared, con la vista fija en un plano horizontal y los pies ligeramente separados, formando una " $V$ ". Por su parte, el peso se midió con cuatro básculas Seca modelo 813, con el sujeto en una postura erguida y con la vista al frente, descalzo, sin chamarra o suéter y sin cargar objetos que modificaran el peso (llaves, bolsas, etc.). ${ }^{36}$ 
Cuadro 1.

\begin{tabular}{|c|c|c|c|c|c|c|c|c|}
\hline & \multicolumn{4}{|c|}{ Mujeres $(n=502)$} & \multicolumn{4}{|c|}{ Hombres $(n=390)$} \\
\hline & Media & DE & mín & máx & Media & DE & mín & máx \\
\hline Peso* $(k g)$ & 58.40 & 10.90 & 35.50 & 103.40 & 70.70 & 13.10 & 44.10 & 120.10 \\
\hline Estatura $^{*}(\mathrm{~m})$ & 1.57 & 0.06 & 1.31 & 1.77 & 1.70 & 0.06 & 1.53 & 1.92 \\
\hline$I M C^{*}$ & 23.50 & 3.90 & 16.60 & 39.00 & 24.10 & 4.00 & 16.00 & 40.50 \\
\hline
\end{tabular}

\section{Procedimiento}

Se trata de un estudio con un diseño transversal analítico en el que se aplicó una encuesta a estudiantes universitarios en sus aulas, previo consentimiento verbal de los profesores titulares. Se invitó a participar de manera voluntaria a todos los estudiantes inscritos en el primer trimestre. A los estudiantes de 18 años o mayores se les proporcionó un consentimiento por escrito, mientras que a los de 17 años o menores se les dio una hoja de asentimiento, junto con una carta informativa para sus padres.

Los facilitadores y antropometristas fueron estudiantes de la carrera de Nutrición Humana y de Psicología de la misma universidad, quienes recibieron capacitación y estandarización antropométrica previa al trabajo de campo.

\section{Análisis estadístico}

Todos los datos fueron analizados según el sexo. En el análisis descriptivo de la población se obtuvieron frecuencias simples y $\chi^{2}$, ya que todas las variables fueron categóricas. Debido a la presencia de tres categorías en la variable de salida (CAR): sin riesgo (CAR-SR), con riesgo moderado (CAR-RM) y con riesgo alto (CAR-RA); el análisis bivariado se realizó utilizando una tabla de contingencia $2 \times 3$. Para medir la interacción del IMC en la asociación de CAR con el resto de las variables, se incluyó un término de interacción del IMC en distintos modelos, uno para cada variable. Finalmente, por medio de un modelo de regresión logística que integró a todas las variables, se estimó la asociación entre CAR y sus covariables. Se consideró como estadísticamente significativo un valor $p<0.05$ y se estimaron intervalos de confianza (IC) al 95.0\%. Para el procesamiento de datos se utilizó el paquete estadístico STATA $11 .^{37}$

\section{RESULTADOS}

\section{Participantes}

La muestra estuvo formada por 892 estudiantes de primer ingreso a la UAM-X, de los cuales 56.2\%(502) fueron mujeres y $43.7 \%$ (390) fueron hombres. No se encontraron diferencias estadísticamente significativas sobre el peso corporal, la estatura o el IMC por sexo (cuadro 1). La edad promedio fue de $19.3 \pm 2.4$ años para las mujeres y $20.4 \pm 3.3$ años para los hombres $(p<0.05)$. Cerca de tres cuartas partes de las mujeres y poco más de la mitad de los varones se concentra en el grupo de edad de 19 años o menos $(p<0.05)$ (cuadro 2).

\section{Análisis descriptivo}

En relación con el NSE, se encontró una mayor proporción de hombres clasificados en el estrato más alto (30.0\%), en comparación con las mujeres $(22.9 \%)(p<0.05)$. Se observa, asimismo, un mayor porcentaje de mujeres en las categorías de NSE más bajas en comparación con los hombres (23.3\% vs. $17.6 \%)$ ( $p<0.05)$. La mitad de las mujeres están inscritas en alguna de las carreras del área de las CBS, en comparación con los hombres (51.2\% y 41.7\%), mientras que un número mayor de varones está inscrito en el área de las CSH. Respecto a la distribución del IMC, 30.0\% de las mujeres y $35.0 \%$ de los hombres presentaron sobrepeso y obesidad $(p>0.05)$. El deseo por una figura más delgada y la IIED estuvieron presentes, respectivamente, en $69.0 \%$ y $11.0 \%$ de las mujeres.

Tanto en hombres como en mujeres, el DM se presentó en $13 \%$, mientras que la baja autoestima se observó en $19 \%$ de las mujeres y $18 \%$ de los hombres. Ambas condiciones no mostraron diferencias estadísticamente significativas entre los sexos $(p>0.005)$ (cuadro 2$)$.

\section{Análisis bivariado}

Poco más de $20.0 \%$ de las mujeres con sobrepeso presentó CAR-RM y 13.2\% CAR-RA. En tanto, para las mujeres con obesidad, las prevalencias fueron $37.8 \%$ y $10.8 \%$ respectivamente $(p<0.05)$. En los hombres con sobrepeso, las prevalencias de CAR-RM y CAR-RA fueron $18.2 \%$ y $3.8 \%$, respectivamente. Las frecuencias de CAR-RM y CAR-RA en hombres con obesidad fueron, respectivamente, 33.3\% y 11.1\% ( $p<$ 0.05). En la categoría peso normal, $17.5 \%$ de las mujeres y $11.9 \%$ de los hombres presentaron CAR-RM. Se estimó que $6.9 \%$ de las mujeres con desnutrición presentaron CAR-RM (cuadro 3). Se estimó que la IIED aumenta el riesgo de CAR 7.4 veces en las mujeres y 5.1 veces en los hombres. Se observa, así mismo, que el DM aumenta el riesgo de CAR 4.4 veces en los hombres y 1.1 veces en las mujeres. 
Cuadro 2. Características generales de la población según sexo

\begin{tabular}{|c|c|c|c|c|}
\hline & $\begin{array}{c}\text { Mujeres } \\
\%(n)\end{array}$ & $\begin{array}{c}\text { Hombres } \\
\%(n)\end{array}$ & $\begin{array}{l}\text { Total } \\
\%(n)\end{array}$ & $p$ \\
\hline \multicolumn{5}{|l|}{ Edad } \\
\hline 19 y menos & $71.3(356)$ & $53.6(208)$ & $63.5(564)$ & $<0.0001$ \\
\hline 20 y más & $28.6(143)$ & 46.3 (180) & 36.4 (323) & \\
\hline \multicolumn{5}{|l|}{ Nivel socioeconómico } \\
\hline$A / B$ & $22.9(115)$ & 30.0 (117) & $26.3(232)$ & 0.0420 \\
\hline $\mathrm{C}_{+}$ & $30.8(155)$ & 31.7 (124) & $31.2(279)$ & \\
\hline C & $30.8(115)$ & $20.5 \quad(80)$ & 21.8 (195) & \\
\hline$D+/ D$ & $23.3(117)$ & $17.6 \quad(69)$ & $20.8(186)$ & \\
\hline \multicolumn{5}{|l|}{ Área de estudio } \\
\hline Ciencias y Artes para el Diseño & 13.4 (67) & $19.4 \quad(75)$ & $16.0(142)$ & 0.0080 \\
\hline Ciencias Sociales y Humanidades & $35.3(176)$ & $38.8(150)$ & $36.8(326)$ & \\
\hline Ciencias Biológicas y de la Salud & $51.2(255)$ & 41.7 (161) & $47.0(416)$ & \\
\hline \multicolumn{5}{|l|}{ Índice de masa corporal } \\
\hline Desnutrición & $5.8 \quad(29)$ & $3.8 \quad(15)$ & $4.9 \quad(44)$ & 0.2070 \\
\hline Normal & 64.2 (320) & $60.2(235)$ & $62.4(555)$ & \\
\hline Sobrepeso & $22.6(113)$ & $26.6(104)$ & $24.3(217)$ & \\
\hline Obesidad & $7.4 \quad(37)$ & $9.2(36)$ & $8.2(73)$ & \\
\hline \multicolumn{5}{|l|}{ Conductas alimentarias de riesgo } \\
\hline Sin riesgo & $74.3(373)$ & 80.7 (315) & 77.1 (688) & 0.0680 \\
\hline Riesgo moderado & $19.5(98)$ & $15.1 \quad(59)$ & $17.6(157)$ & \\
\hline Riesgo alto & 6.8 (31) & $4.1 \quad(16)$ & 5.2 (47) & \\
\hline \multicolumn{5}{|l|}{ Insatisfacción corporal } \\
\hline Sin insatisfacción & $21.1(106)$ & $29.4(115)$ & $24.8(221)$ & $<0.0001$ \\
\hline Insatisfacción: desea figura más gruesa & 9.3 (47) & $31.2(122)$ & 18.9 (169) & \\
\hline Insatisfacción: desea figura más delgada & 69.4 (348) & $39.2(153)$ & $56.2(501)$ & \\
\hline \multicolumn{5}{|l|}{ Interiorización del ideal estético de delgadez } \\
\hline Sí & 11.3 (57) & 6.9 (27) & $9.4 \quad(84)$ & 0.0250 \\
\hline No & $88.6(445)$ & $93.0(363)$ & $90.5(808)$ & \\
\hline \multicolumn{5}{|l|}{ Deseo por la musculatura } \\
\hline Sí & 13.3 (67) & $13.8 \quad(54)$ & 13.5 (121) & 0.8290 \\
\hline No & 86.6 (435) & 86.6 (336) & 86.4 (771) & \\
\hline \multicolumn{5}{|l|}{ Baja autoestima } \\
\hline Sí & $19.5 \quad(98)$ & $18.4 \quad(72)$ & 19.0 (170) & 0.6890 \\
\hline No & 80.4 (404) & 81.5 (318) & $80.0(722)$ & \\
\hline
\end{tabular}

Los resultados muestran que la baja autoestima aumenta el riesgo de CAR 2 veces, mientras que los síntomas depresivos lo hacen en 2.3 veces exclusivamente en el grupo de mujeres. Respecto a la insatisfacción corporal, se encontró que únicamente las mujeres y hombres que desean una figura más delgada tienen riesgo de CAR (3.6 veces y 2.7 veces respectivamente). El IMC aumenta el riesgo de CAR 1.6 veces en mujeres $(p<0.05)$ (cuadro 4$)$.

\section{Análisis multivariado}

Cuando se analizó la interacción del IMC en la asociación de CAR con cada una de las variables, no se encontraron valores estadísticamente significativos. Por lo tanto, el IMC se incluyó en el modelo de regresión como una variable independiente o explicativa. La categoría de NSE D+/D tiene un efecto protector para CAR sólo en el grupo de hombres. Así mismo, se observa exclusivamente en los varones que las áreas de estudio asociadas con un mayor riesgo para CAR son CSH y CBS (cuadro 4).

\section{DISCUSIÓN Y CONCLUSIÓN}

Como resultados principales, se encontró que las CAR están presentes tanto en hombres como en mujeres, sin diferencias estadísticamente significativas. Esto difiere de la idea prevaleciente acerca de que las CAR y los TCA son condiciones privativas o mayoritariamente un problema de las mujeres, lo cual nos obliga a replantear dicha idea, pero también a dirigir los esfuerzos por informar y prevenir los TCA en poblaciones de ambos sexos. En virtud de lo anterior se puede inferir un subregistro de los casos de TCA en hombres, atribuible a que éstos no acuden a tratamiento con la frecuencia en que lo hacen las mujeres, ya sea porque no se consideran como enfermedades de varones, porque los síntomas no 
Cuadro 3. Conductas alimentarias de riesgo por sexo, según IMC

\begin{tabular}{|c|c|c|c|c|c|c|c|c|}
\hline \multirow[b]{3}{*}{ IMC } & \multicolumn{8}{|c|}{ Conductas alimentarias de riesgo } \\
\hline & \multicolumn{4}{|c|}{ Mujeres } & \multicolumn{4}{|c|}{ Hombres } \\
\hline & $\begin{array}{c}\text { Sin riesgo } \\
\%(n)\end{array}$ & $\begin{array}{c}\text { Riesgo moderado } \\
\%(n)\end{array}$ & $\begin{array}{c}\text { Riesgo alto } \\
\%(n)\end{array}$ & $p$ & $\begin{array}{c}\text { Sin riesgo } \\
\%(n)\end{array}$ & $\begin{array}{c}\text { Riesgo moderado } \\
\%(n)\end{array}$ & $\begin{array}{l}\text { Riesgo alto } \\
\%(n)\end{array}$ & $p$ \\
\hline Desnutrición & $93.1 \quad(27)$ & $6.9(2)$ & $0.0(0)$ & $<0.0010$ & $100.0(15)$ & $0.0(0)$ & $0.0(0)$ & $<0.0001$ \\
\hline Normal & $78.7(252)$ & $17.5(56)$ & $3.7(12)$ & & 84.6 (199) & $11.9(28)$ & $3.4(8)$ & \\
\hline Sobrepeso & $64.6(73)$ & $22.1(25)$ & $13.2(18)$ & & $77.8(81)$ & 18.2 (19) & 3.8 (4) & \\
\hline Obesidad & $51.3(19)$ & $37.8(14)$ & $10.8(4)$ & & $55.5(20)$ & $33.3(12)$ & $11.1(5)$ & \\
\hline
\end{tabular}

evolucionan hasta requerir tratamiento especializado o porque no se identifican como un problema de salud.

Los resultados mostraron también que existe riesgo para desarrollar CAR en las mujeres cuando se ha interiorizado el IIED; se desea un cuerpo musculoso, se presentan síntomas depresivos, se desea de una figura corporal más delgada y se presenta un mayor IMC. Por su parte, en los hombres, las variables que mostraron riesgo fueron la IIED, el DM, el deseo de una figura corporal más delgada y estudiar una carrera de las áreas de estudio CBS y $\mathrm{CSH} .{ }^{19,20,38}$

A pesar de la ausencia de datos nacionales sobre CAR en población del mismo grupo de edad, consideramos que para dar una idea de la problemática en el país, la comparación con los datos de la ENSANUT $2012^{5}$ aporta información relevante. Los resultados de este trabajo mostraron que hay mayor frecuencia de CAR entre los estudiantes de la muestra en comparación con una población abierta de ado- lescentes de México ( $0.8 \%$ en hombres y $1.9 \%$ en mujeres). Esto supone un mayor riesgo de TCA en estudiantes universitarios que entre la población abierta; no obstante, que en esta muestra los porcentajes encontrados en mujeres fueron menores, en comparación con otros estudios realizados en México. ${ }^{11,12}$

El IMC ha sido asociado anteriormente con la frecuencia de CAR en diferentes investigaciones en México. ${ }^{8,39-41}$ En este estudio, se encontró una prevalencia menor de sobrepeso y obesidad en los jóvenes participantes en comparación con los datos nacionales reportados en la ENSANUT-2012, ${ }^{5}$ pero un porcentaje similar de CAR.

Se encontró una relación positiva entre el IMC y la frecuencia de las CAR, y la ausencia de interacción del IMC en la asociación de CAR con el resto de las variables. Tales hallazgos coinciden con lo reportado por Unikel et al., ${ }^{39}$ quienes también reportaron un aumento en la frecuencia de CAR cuando aumenta el IMC en mujeres entre 15 y 19 años,

Cuadro 4. Análisis de regresión, según sexo

\begin{tabular}{|c|c|c|c|c|c|c|c|c|}
\hline \multirow[b]{3}{*}{ Variable } & \multicolumn{4}{|c|}{ Mujeres } & \multicolumn{4}{|c|}{ Hombres } \\
\hline & \multirow[t]{2}{*}{ Riesgo } & \multirow[t]{2}{*}{$p$} & \multicolumn{2}{|c|}{ IC 95\% } & \multirow[t]{2}{*}{ Riesgo } & \multirow[t]{2}{*}{$p$} & \multicolumn{2}{|c|}{ IC 95\% } \\
\hline & & & Mín & Máx & & & Mín & Máx \\
\hline $\begin{array}{l}\text { Interiorización del ideal estético } \\
\text { de delgadez }\end{array}$ & 7.40 & $<0.0001$ & 3.8593 & 14.5009 & 5.1 & $<0.0001$ & 2.1071 & 12.6992 \\
\hline Deseo por la musculatura & 1.10 & 0.643 & 0.5916 & 2.3389 & 4.4 & $<0.0001$ & 2.1893 & 9.1674 \\
\hline Autoestima & 2.00 & 0.017 & 1.1336 & 3.5851 & 1.9 & 0.063 & 0.9641 & 3.8234 \\
\hline $\begin{array}{l}\text { Síntomas de depresión } \\
\text { Insatisfacción corporal: }\end{array}$ & 2.30 & 0.006 & 1.2771 & 4.2924 & 1.8 & 0.162 & 0.7853 & 4.2891 \\
\hline Desea figura más gruesa & $<0.01$ & 0.980 & - & - & 0.6 & 0.427 & 0.2660 & 1.7512 \\
\hline Desea figura más delgada & 3.60 & 0.002 & 1.5840 & 8.5321 & 2.7 & 0.009 & 1.2982 & 6.0209 \\
\hline IMC & 1.60 & 0.006 & 1.1546 & 2.3069 & 1.4 & 0.124 & 0.9105 & 2.1880 \\
\hline Edad & 0.79 & 0.402 & 0.4704 & 1.3527 & 1.7 & 0.065 & 0.9653 & 3.2816 \\
\hline \multicolumn{9}{|l|}{ Nivel socioeconómico* } \\
\hline $\mathrm{C}+$ & 0.90 & 0.921 & 0.5195 & 1.8075 & 0.5 & 0.138 & 0.2879 & 1.1887 \\
\hline $\mathrm{C}$ & 1.20 & 0.517 & 0.6418 & 2.4151 & 0.6 & 0.280 & 0.2829 & 1.4410 \\
\hline$D+/ D$ & 0.60 & 0.227 & 0.3039 & 1.3262 & 0.3 & 0.028 & 0.1233 & 0.8845 \\
\hline \multicolumn{9}{|l|}{ Área de estudio** } \\
\hline $\mathrm{CSH}$ & 0.50 & 0.052 & 0.2516 & 1.0056 & 2.9 & 0.037 & 1.0693 & 8.0679 \\
\hline CBS & 0.50 & 0.071 & 0.2800 & 1.0559 & 3.8 & 0.010 & 1.3825 & 10.6762 \\
\hline
\end{tabular}

$\mathrm{CSH}=$ Ciencias Sociales y Humanidades; $\mathrm{CBS}=$ Ciencias Biológicas y de la Salud; C+ = nivel socioeconómico medio alto; $\mathrm{C}=$ nivel socioeconómico medio; $D+/ D=$ nivel socioeconómico bajo medio y bajo. ${ }^{*}$ Grupo de referencia $=A / B$, el nivel socioeconómico más alto. ${ }^{*}$ Grupo de referencia $=$ Ciencias y Artes para el Diseño. 
así como la ausencia de interacción entre variables como crítica e IMC sobre la frecuencia de CAR. Este aumento en la frecuencia de CAR asociado con el aumento de IMC también fue reportado por Lora-Cortez y Saucedo-Molina ${ }^{40}$ en una muestra de mujeres entre 25 y 45 años.

Cada una de las variables impacta de manera individual a las CAR, independientemente del IMC. Sin embargo, en las mujeres, ésta última variable incrementa el riesgo de CAR de manera independiente. Se puede pensar que las mujeres están más inconformes con un cuerpo más grande que los hombres y que la presión por la delgadez es mayor en ellas, ${ }^{42}$ por lo cual se espera que, a mayor IMC, se muestre no sólo mayor insatisfacción con el peso y la figura, sino también más CAR.

Un hallazgo importante tiene que ver con un cambio observado en el ideal de belleza femenino, en el cual, además de un cuerpo delgado, se pide como requisito que esté tonificado, por lo que se entiende el resultado obtenido en cuanto al DM en las mujeres de la muestra.

En los hombres se observó una asociación entre las CAR y el área de estudio, siendo las áreas de mayor riesgo $\mathrm{CSH}$ y CBS. Esta relación se ha encontrado con anterioridad en estudios realizados en poblaciones de deportistas, donde fueron reportados con mayor riesgo los estudiantes de alguna carrera del área de la salud. ${ }^{43}$ En México, se ha encontrado que los hombres estudiantes de Nutrición presentan mayor prevalencia de CAR que las mujeres estudiantes de la misma carrera. ${ }^{13}$ Blanco-Fernández, ${ }^{44}$ encontró una relación entre la selección de carrera y TCA, proponiendo como explicación que los sujetos que escogen carreras relacionadas con las áreas de la salud, la comunicación o los deportes, dan mucho valor a la estética corporal, al impacto de los medios de comunicación y a la educación.

Algunas limitaciones del estudio son que, debido a que se trata de un estudio transversal, no se puede demostrar causalidad en las relaciones entre variables, y si bien, se trata de una muestra representativa de los estudiantes de primer ingreso a la UAM, sólo es generalizable a esta población de estudiantes en México. Los cuestionarios de auto-reporte implican un sesgo en la investigación debido a las fallas en la memoria y a la deseabilidad social. Por su parte, la medición de las CAR y la IIED en hombres puede tener un subregistro debido a que el cuestionario utilizado fue desarrollado en una muestra de mujeres.

No obstante las limitaciones mencionadas, se lograron los objetivos de este trabajo: analizar la relación entre CAR e IMC en una muestra de estudiantes universitarios; determinar la asociación de las CAR con variables demográficas (NSE, edad y área de estudio) y psicológicas (IIED, IC, autoestima, DM), y conocer la interacción del IMC con dichas asociaciones. Además, esta investigación aporta datos importantes que ayudan a profundizar el conocimiento de las CAR en la población universitaria mexicana y permite comprender mejor las necesidades que habría que atender en relación con esta problemática en los estudiantes de nivel superior.

\section{Financiamiento}

Ninguno.

\section{Conflicto de intereses}

Los autores declaran no tener ningún conflicto de intereses.

\section{REFERENCIAS}

1. Hershner SD, Chervin RD. Causes and consequences of sleepiness among college students. Nat Sci Sleep 2014; 6:73-84.

2. Nyer M, Farabaugh A, Fehlin K, Soskin D et al. Relationship between sleep disturbance and depression, anxiety, and functioning in college students. Depress Anxiety 2013;30:doi:10.1002/da.22064.

3. Rivera-Márquez JA, Díaz de León-Vázquez C, Oropeza-Aguirre MF, Unikel-Santoncini C. Conductas alimentarias de riesgo en estudiantes de la Universidad Autónoma Metropolitana: diagnósticos preliminares. En: Lara-Flores NL, Eibenschutz-Hartman C, Tamez-González S, Ehrenfeld-Lenkiewicz N (eds.). La salud y su atención: Problemas actuales, miradas diversas. México, D.F., UAM, 2013:177-198. ISBN: 978607-28-0128-8.

4. Organización Mundial de la Salud (OMS). Serie de Informes Técnicos, No 916. Dieta, nutrición y prevención de enfermedades crónicas. Informe de un grupo de estudio de la OMS. Ginebra: 2002.

5. Gutiérrez JP, Rivera-Dommarco J, Shamah-Levy T, Villalpando-Hernández $S$ et al. Encuesta Nacional de Salud y Nutrición 2012. Resultados nacionales. Cuernavaca, México: Instituto Nacional de Salud Pública; 2012.

6. Saguy AC, Riley KW. J Health Politics, Policy Law. 2005; 30(5): 869-923. doi: 10.1215/03616878-30-5-869.

7. Newmark-Sztainer D, Wall M, Haines J, Story M, Eisenberg ME. Why does dieting predict weight gain in adolescents? Findings fron proyect EAT-II: A 5-year longitudinal study. J Am Diet Assoc 2007;107:448-455.

8. Unikel-Santoncini C, Saucedo-Molina T, Villatoro J, Fleiz C. Conductas alimentarias de riesgo y distribución del índice de masa corporal en estudiantes de 13 a 18 años. Salud Ment 2002;25:49-57.

9. Stice E. Risk factors for eating pathology: recent advances and future directions. En: Eating disorders: innovative directions in research and practice. Washington: American Psychological Association; 2001; pp.51-73.

10. Palma-Coca O, Hernández-Serrato MI, Villalobos-Hernández A, Unikel-Santoncini $\mathrm{C}$ et al. Association of socioeconomic status, problem behaviors, and disordered eating in Mexican adolescents: Results of the Mexican national health and Nutrition Survey 2006. J Adolesc Health 2011;49:400-406.

11. Saucedo-Molina TJ, Unikel-Santoncini C. Conductas alimentarias de riesgo, interiorización del ideal estético de delgadez e índice de masa corporal en estudiantes hidalguenses de preparatoria y licenciatura de una institución privada. Salud ment 2010;33:11-19.

12. Álvarez-Rayón G, Franco-Paredes K, Vázquez-Arévalo R, Escarria-Rodríguez AR et al. Sintomatología de trastorno alimentario: comparación entre hombres y mujeres estudiantes. Psicología Salud 2003;13:245-255.

13. Cruz-Bojórquez RM, Ávila Escalante ML, Cortés-Sobrino MC, Vázquez-Arévalo $\mathrm{R}$ et al. Restricción alimentaria y conductas de riesgo de trastornos alimentarios en estudiantes de nutrición. Psicología Salud 2008;18:189-198.

14. Costa LCF, Guedes de Vasconcelos FA, Glazer-Peres K. Influence of Biological, Social and Psychological Factors on Abnormal Eating At- 
titudes among Female University Students in Brazil. J Health Popul Nutr 2010;28:173-181.

15. Fandiño A, Giraldo SC, Martínez C, Aux CP et al. Factores asociados con los trastornos de la conducta alimentaria en estudiantes universitarios de Calí, Colombia. Colombia Médica 2007;38(4):344-351.

16. Lugli-Rivero Z, Vivas-Peñalver E. Conductas y actitudes sugestivas de trastornos del comportamiento alimentario en estudiantes universitarios venezolanos. Revista Facultad Medicina (Caracas) 2006;29(2):168173.

17. Tiggemann M. Objectification theory: Of relevance for eating disorder researchers and clinicians? Clin Psychologist 2013;17:35-45.

18. Cafri G, Thompson KJ, Ricciardelli L, McCabe M et al. Pursuit of the muscular ideal: Physical and psychological consequences and putative risk factors. Clin Psychol Rev 2005;25:215-239.

19. Eisenberg D, Nicklett EJ, Roeder K, Kirz NE. Eating disorder symptoms among college students: Prevalence, persistence, correlates, and treatment-seeking. J Am Coll Health 2011;59:700-707.

20. Jacobi C, Fittig E, Bryson SW, Wilfley D et al. Who is really at risk? Identifying risk factors for subthreshold and full syndrome eating disorders in a high-risk simple. Psychol Med 2011;41:1939-1949.

21. Stice E, Ng J, Shaw $\mathrm{H}$. Risk factors and prodromal eating pathology. J Child Psychol Psychiat 2010;51:518-525.

22. Jacobi C, Hayward C, de Zwaan, Kraemer HC et al. Coming to terms with risk factors for eating disorders: application of risk terminology and suggestions for a general taxonomy. Psychol Bull 2004;13:19-65.

23. López-Romo H. Asociación Mexicana de Agencias de Investigación de Mercado y Opinión Publica A.C. AMAI [sede web]. Índice AMAI. Regla AMAI 10x6. Los niveles socioeconómicos y la distribución del gasto. Instituto de Investigaciones Sociales SC. 2009. Disponible en: www.amai.org/NSE/NivelSocioeconomicoAMAI.pdf.

24. Unikel-Santoncini C, Bojorquez-Chapela I, Carreño-García S. Validación de un cuestionario breve para medir conductas alimentarias de riesgo. Salud Púb Méx 2004;46:509-515.

25. Unikel C, Juárez F, Gómez Peresmitré G. Psychometric properties of the attitudes towards body figure questionnaire in female students and patients with eating disorders. Eur Eat Disorder Rev 2006;14:430435.

26. Pope AW, McHale SM, Craighead WE. Self-esteem enhancement with children and adolescents. USA: Allyn \& Bacon Eds; 1998.

27. Unikel-Santoncini C, Gómez-Peresmitré G. Validez de constructo de un instrumento para la detección de factores de riesgo en los trastornos de la conducta alimentaria en mujeres mexicanas. Salud Ment 2004;27:38-49.

28. Eaton W, Muntaner C, Smith C. Revision of the Center of Epidemiological Studies Depression (CES-D) Scale. Baltimore: Johns Hopkins University Prevention Center; 1998.

29. González-Forteza C, Jiménez-Tapia JA, Ramos-Lira L, Wagner FA. Aplicación de la Escala de Depresión del Center of Epidemiological Studies en adolescentes de la Ciudad de México. Salud Publica Mex 2008;50:292-299.
30. McCreary D, Sasse D. An exploration of the drive for muscularity in adolescent boys and girls. J Am Coll Health 2000;48:297-304.

31. Escoto C, Álvarez-Rayón G, Mancilla-Díaz JM, Camacho Ruiz E et al. Psychometric properties of the Drive for Muscularity Scale in Mexican males. Eat Weight Disord 2013; doi 10.1007/s40519-013-0010-6.

32. Stunkard AJ, Sorensen T, Schulsinger F. Use of the Danish adoption register for the study of obesity and thinness. En: Kety $S$ (ed.). The genetics of neurological and psychiatric disorders. New York: Raven Press; 1983.

33. Acosta-García MV, Gómez-Peresmitré G. Insatisfacción corporal y seguimiento de dieta: una comparación transcultural entre adolescentes de España y México. Int J Clin Health Psychol 2003;3:9-21.

34. Physical Activity Guidelines for Americans. U.S. Department of Health and Human Services [sede web]. [Actualizado: 16 de octubre de 2008] Disponible en: www.health.gov/paguidelines/guidelines/Chapter3 y www.health.gov/paguidelines/guidelines/Chapter4

35. Organización Mundial de la Salud (OMS). WHO [sede web]. Fecha de actualización: 22 de agosto 2013; fecha de acceso: 22 de agosto 2013. Global data base on body mass index. BMI classification. Disponible en: http://apps.who.int/bmi/index.jsp?introPage=intro_3.htm.

36. Shamah-Levy T, Villalpando- Hernández S, Rivera-Dommarco J. Manual de Procedimientos para Proyectos de Nutrición. Cuernavaca, México: Instituto Nacional de Salud Pública; 2006.

37. StataCorp. Stata Statistical Software: Release 10. College Station, TX: StataCorp LP; 2007.

38. Thompson KJ, Stice E. Thin-ideal internalization: mounting evidence of a new risk factor for body-image disturbance and eating pathology. Current Directions Psychol Sci 2001;10:181-183.

39. Unikel-Santoncini C, Martín-Martín V, Juárez-García F, González-Forteza $C$ et al. Disordered eating behavior and body weight and shape: Relatives' criticism in overweight and obese 15- to 19-year-old females. J Health Psychol 2013;18:75-85.

40. Altamirano-Martínez MB, Bízmanos-Lamotte B, Unikel-Santoncini C. Continuo de conductas alimentarias de riesgo en adolescentes de México. Rev Panam Salud Pub 2011;30:401-407.

41. Lora-Cortez CI, Saucedo-Molina TJ. Conductas alimentarias de riesgo e imagen corporal de acuerdo al índice de masa corporal en una muestra de mujeres adultas de la Ciudad de México. Salud Ment 2006;29:6067.

42. Vaquero-Cristóbal R, Alacid F, Muyor JM, López-Miñarro PA. Imagen corporal; revisión bibliográfica. Nutr Hosp 2013;28:27-35.

43. Vásquez R, Ángel Arango LA, García Valencia J, Martínez Gil LM et al. Trastornos de la conducta alimentaria en deportistas. Rev Col Psiquiatría 2003;26:115-126.

44. Blanco Fernández MA. "Los trastornos de la ingesta hoy" estudio de una muestra de estudiantes universitarios . I Congreso Virtual de Psiquiatría 1 de febrero-15 de marzo 2000 [citado: 1 de junio de 2015]; Conferencia 24-CI-H: [14 pantallas]. Disponible en: http://www.psiquiatria.com/congreso/mesas/mesa24/conferencias/24_ci_h.htm 